\title{
Triangles in squares
}

Richard P. Jerrard and John E. Wetzel

\begin{abstract}
After army service and work in engineering, Richard Jerrard received his Ph.D. from the University of Michigan in 1957. He worked first in applied mathematics, then shifted to topology and geometry. At the University of Illinois since 1958, he spent several years at the University of Warwick and Cambridge University. He retired in 1995.

John Wetzel received his Ph.D. in mathematics from Stanford University in 1962, a student of Halsey Royden. He retired in 1999 from the University of Illinois in Urbana, after 38 years of service. Always interested in classical geometry, he has most recently been studying the ways in which one shape fits in another - questions he regards as "fitting problems for retirement."
\end{abstract}

Introduction. In this note we determine precisely when a triangle fits in a square by finding necessary and sufficient conditions on the sides $a, b, c, s$ for the triangle with sides $a, b, c$ to fit into the square of side $s$. Our strategy is to find the side $s_{\min }(T)$ of the smallest square $S_{\min }$ that contains the given triangle $T$; then $T$ fits into a square $S$ of side $s$ precisely when $s \geq S_{\min }(T)$. Scaling solves the equivalent dual problem: Find the largest triangle similar to a given triangle that fits in a given square.

Minimal squares about a triangle. By a square we sometimes mean the union of four line segments and sometimes the region they surround - the precise meaning will always be clear from the context. A triangle has vertices while a square has corners, and unless

Die Frage nach den Bedingungen, unter denen eine geometrische Figur in eine andere einbeschrieben werden kann, ist auch heute noch aktuell. Dies belegt zum Beispiel der Artikel von K.A. Post aus den neunziger Jahren, in dem eine Lösung zu einer alten Fragestellung von Steinhaus gegeben wird, notwendige und hinreichende Bedingungen für die sechs Seiten zweier Dreiecke zu finden, so dass das eine Dreieck in das andere einbeschrieben werden kann. In dem vorliegenden Artikel untersuchen die Autoren, wann ein Dreieck in ein Quadrat einbeschrieben werden kann. Dazu geben sie notwendige und hinreichende Bedingungen an, die die drei Dreiecksseiten und die Quadratseite zu erfüllen haben. Die duale, klassische Problemstellung, wann nämlich ein Quadrat in ein Dreieck einbeschrieben werden kann, hat einer der Autoren im stumpfwinkligen Fall vor kurzem studiert. 
the contrary is explicitly stated, a side of either includes its ends. It is a consequence of compactness that among all squares that contain a given triangle $T$ there is at least one whose side is as small as possible. We begin by considering how such a minimal square fits about the given triangle.

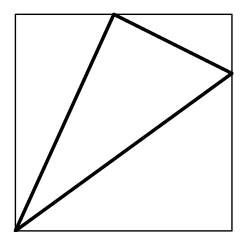

(a) Vertex square

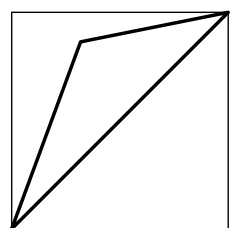

(b) Diagonal square

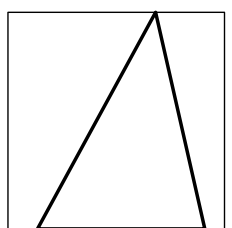

(c) Side square

Fig. 1 Minimal squares

The possibilities are pictured in Fig. 1. A vertex square of $T$ is a square containing $T$ with exactly one vertex of $T$ at a corner of the square and the other two vertices on the two (open) non-adjacent sides of the square (Fig. 1(a)). A diagonal square of $T$ is a square containing $T$ whose diagonal is a side of $T$ (Fig. 1(b)). A side square of $T$ is a non-diagonal square that contains $T$ with two vertices on one side and the third vertex on the opposite side (Fig. 1(c)). We call all such squares minimal.

Lemma 1 The smallest square $S_{\min }$ that contains a given triangle $T$ is a vertex, a diagonal, or a side square of $T$.

Proof. Suppose $S_{\min }$ is not a diagonal square of $T$. Then all three vertices of $T$ must lie on $S_{\min }$ (otherwise a suitable rigid motion would move $T$ entirely into the interior of $S_{\min }$, and it would lie in a square strictly smaller than $S_{\min }$ ). We examine the possibilities for the three vertices on the four sides of $S_{\min }$. If two vertices are on one side and the third is on an adjacent side, then $T$ could be moved into the interior of $S_{\min }$ by a suitable rigid motion, a contradiction. If two vertices are on one side and the third is on the opposite side, then the square is a side square. If no two vertices of $T$ are on the same side of $S_{\min }$ and no vertex lies at a corner of $S_{\min }$, then $T$ could be moved into the interior of $S_{\min }$ by a suitable rigid motion, again a contradiction. The only remaining possibility is that $S_{\min }$ is a vertex square.

We will find that if two angles of $T$ are less than or equal to $45^{\circ}$, then the smallest square $S_{\min }$ is the diagonal square on the longest side, and in every other situation with just one exception, $S_{\min }$ is a vertex square. The exception, in which $S_{\min }$ is the side square on the shortest side of $T$, occurs when $T$ is acute, the altitude to the shortest side is longer than that side, and the smallest angle is greater than $45^{\circ}$.

The principal results. In this section we determine precisely when squares of each kind exist and which is smallest when there is more than one.

Let $T=A B C$, and write $\alpha, \beta, \gamma$ for $\angle A, \angle B, \angle C$, respectively. For $\{X, Y, Z\}=$ $\{A, B, C\}$ we write $S_{X}, S_{X Y}$, and $S_{x}$ for the vertex square at the vertex $X$, the diagonal square on the side $X Y$, and the side square on the side $x$ opposite the vertex $X$, respectively, when they exist. We denote by $h_{x}$ the altitude of $T$ to the side $x$. 
Vertex squares. A vertex square can exist only at a vertex of $T$ where the angle is acute. We begin by determining precisely when there is a vertex square at such a vertex.

Lemma 2 Suppose that $T=A B C$ with $\gamma<90^{\circ}$. Then there is a vertex square at $C$ if and only if both

$$
h_{a}<a \quad \text { and } \quad h_{b}<b .
$$

The square is unique when it exists, and its side s is given by the formula

$$
s=\frac{a b \cos \gamma}{\sqrt{a^{2}-2 a b \sin \gamma+b^{2}}} .
$$

Proof. Suppose such a square $C P Q R$ exists (Fig. 2), and let $L$ be the foot of the perpendicular from $B$ to $C P$. Suppose the altitude ray $B E$ meets $C P$ at $Y$. Then $\angle P C A=\angle L B Y$, the right triangles $C P A$ and $B L Y$ are congruent, and $B Y=C A=b$. Since $E$ lies between $B$ and $Y$ (because $A$ lies between $P$ and $Q$ ), $h_{b}=B E<B Y=b$. A similar argument on the altitude ray $A D$ shows that $h_{a}<a$, so that conditions (1) are necessary. When the vertex square $S_{C}=C P Q R$ exists,

$$
\tan \angle P C A=\frac{E Y}{C E}=\frac{B Y-B E}{C E}=\frac{b-a \sin \gamma}{a \cos \gamma},
$$

which establishes the uniqueness. Formula (2) for the side $s$ follows from substituting (3) into $s=b \cos \angle P C A$.

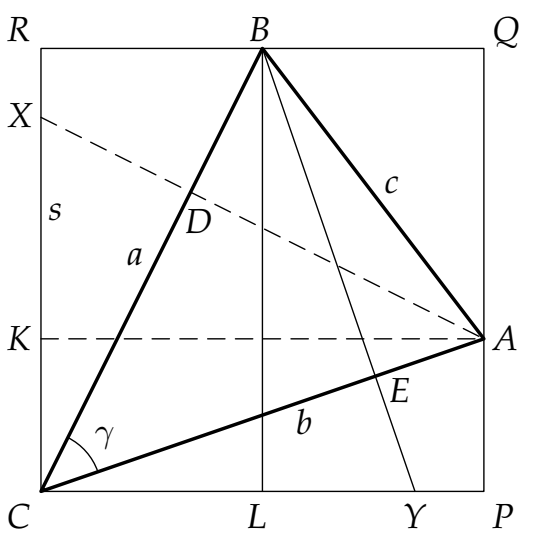

Fig. 2 Vertex square

To show the converse, take $Y$ on the altitude ray $B E$ so that $B Y=b$, and let $P$ be the foot of the perpendicular from $A$ to the ray $C Y$. Let $L$ be the foot of the perpendicular from $B$ to $C Y$. Then $\angle P C A=\angle L B Y$, triangles $C P A$ and $B L Y$ are congruent, and $B L=C P$. Take points $Q, R$ so that the figure $C P Q R$ is a square. According to the hypothesis $h_{b}<b, Y$ and $B$ are on opposite sides of $C A$ and $A$ lies between $P$ and $Q$. It remains only to show that $B$ lies between $Q$ and $R$. Suppose the altitude ray $A D$ from 
$A$ to $B C$ meets $C R$ at the point $X$, and let $K$ be the foot of the perpendicular from $A$ to $C R$. Then $\angle X A K=\angle B C R$, and it follows that right triangles $R C B$ and $K A X$ are congruent and $A X=C B=a$. From the hypothesis $h_{a}<a$ we see that $D$ lies between $A$ and $X$, and consequently $B$ lies between $R$ and $Q$.

The argument of the proof gives an elegant ruler and compass construction for the vertex square $C P Q R$ when it exists.

If $T$ has two vertex squares, say one at $B$ and one at $C$, which is smaller? We show next that the smaller square is at the vertex whose angle is larger.

Lemma 3 Suppose that $T$ has vertex squares $S_{B}$ at $B$ and $S_{C}$ at $C$. Then $S_{B}$ is larger than, equal to, or smaller than $S_{C}$ according as $\beta<\gamma, \beta=\gamma$, or $\beta>\gamma$.

Proof. Write $s, t$ for the sides of $S_{B}, S_{C}$, respectively, and let $\varphi=\angle P B C$ and $\psi=\angle B C Z$ (Fig. 3). Then $s=a \cos \varphi$ and $t=a \cos \psi$, so that $s<t, s=t$, or $s>t$ according as $\varphi>$ $\psi, \varphi=\psi$, or $\varphi<\psi$. But $\tan \varphi=(a-c \sin \beta) / c \cos \beta$ and $\tan \psi=(a-b \sin \gamma) / b \cos \gamma$ (by (3)). Both parenthetical factors below being 1 by the law of sines, we see that

$$
\frac{\tan \varphi}{\tan \psi}=\left(\frac{a-c \sin \beta}{a-b \sin \gamma}\right) \frac{b \cos \gamma}{c \cos \beta}=\frac{\tan \beta}{\tan \gamma}\left(\frac{b \sin \gamma}{c \sin \beta}\right)=\frac{\tan \beta}{\tan \gamma},
$$

and the conclusion follows.

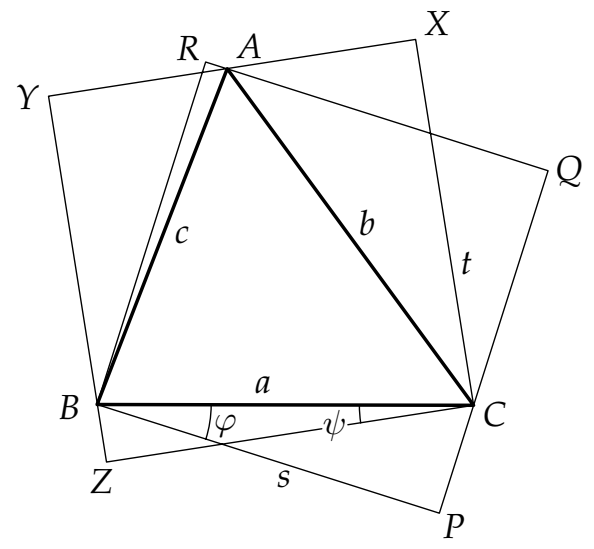

Fig. 3 Two vertex squares

Diagonal squares. The situation in which the smallest square is a diagonal square (Fig. 1(b)) is easy to characterize.

Lemma 4 Suppose $T=A B C$ and $\beta, \gamma \leq 45^{\circ}$. Then the smallest square $S_{\min }$ that contains $T$ is the diagonal square $S_{B C}$ with side $a / \sqrt{2}$. 
Proof. Triangle $T$ fits in $S_{B C}$ as pictured in Fig. 1(b), and since $\operatorname{diam}(T)=a=\operatorname{diam}\left(S_{\min }\right)$, no smaller square will do.

Side squares. When $T$ has a side square (Fig. 1(c)) on a side $x$, plainly $h_{x} \geq x$. It is convenient to introduce a term for this situation: We call a triangle $T$ tall if it has a side $x$ for which $h_{x} \geq x$. So a triangle has a side square only when it is tall.

Lemma 5 If $x, y$ are sides of a non-right triangle and $x \leq y$, then $h_{y}<y$. A right triangle is tall on its shorter leg, and tall on both legs only if it is isosceles.

Proof. If $\theta$ is the angle between sides $x$ and $y$ and $\theta \neq 90^{\circ}$, then $h_{y}=x \sin \theta<x \leq y$. For the legs $x, y$ of a right triangle we have $h_{x}=y$ and $h_{y}=x$, and the result follows.

So the inequality $h_{x} \geq x$ is possible for a triangle that is not isosceles-right only when that triangle has a strictly shortest side, and $x$ is that side.

It is convenient to employ notation that identifies the shortest side of $T$. We agree to label the vertices of $T$ so that $T$ is positively oriented (i.e., the sequence $A \rightarrow B \rightarrow C \rightarrow A$ is counterclockwise) and $\alpha \geq \beta \geq \gamma$, by replacing $T$ by its mirror image if necessary. Then $T$ can be realized in the coordinate plane with $B C$ horizontal and $A$ above the line $B C$; and because of the notational normalization, $A$ lies in the circular triangle $M D B$ bounded by the mediator $M D$ of $B C$ and the arc $D B$ of the circle of radius $a$ centered at C (Fig. 4).

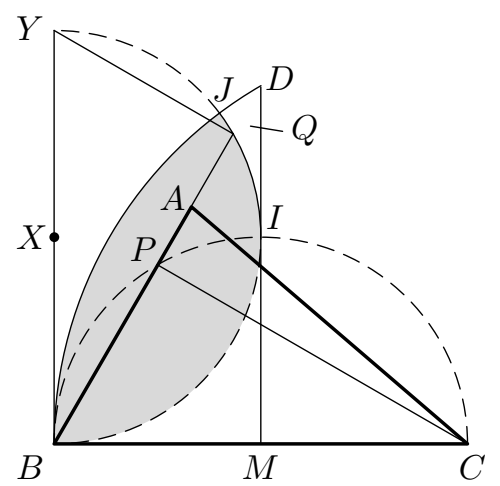

Fig. 4 The tall region

Suppose the semicircle with diameter $B C$ meets the mediator $M D$ at the point $I$. Let $B M I X$ be the square on $B M$, and suppose the circle with radius $a / 2$ centered at $X$ meets the arc $B D$ at $J$ and the ray $B X$ at $Y$ (Fig. 4). We call the lens $B J$ between the two circular arcs, shaded in Fig. 4, the tall region for $T$.

Lemma 6 A triangle $T=A B C$ with $\alpha \geq \beta \geq \gamma$ is tall if and only if $A$ lies in the tall region $B J$; and then $h_{c} \geq c$, with equality precisely when $A$ lies on the circular arc BIJ. 
Proof. Let $A$ lie in the tall region $B J$, and suppose the ray $B A$ meets the semicircle $C I B$ at $P$ and the semicircle $B I Y$ at $Q$ (Fig. 4). Then right triangles $B C P$ and $Y B Q$ are congruent (indeed, a clockwise quarter-turn about $I$ carries the first to the second), and it follows that $B Q=C P=h_{c}$. Consequently, $h_{c}>c, h_{c}=c$, or $h_{c}<c$ according as $A$ lies on the (open) segment $B Q$, at $Q$, or beyond $Q$ on the ray $B P$.

Consequently a triangle (not isosceles right) has a side square if and only if it is tall. That side square is unique when it exists, it rests on the strictly shortest side of $T$, and its side is the longest altitude of $T$.

Although a tall obtuse triangle $T$ has a side square $S_{c}$, that square can never be $S_{\text {min }}$ because $T$ can be moved into its interior by a suitable small motion. When $T$ is tall and not obtuse, then in addition to a side square it also has a vertex square. We need to determine which of these two minimal squares is smaller.

Lemma 7 Let $T=A B C$ be an acute or right triangle (but not isosceles right) with $h_{c} \geq c$. Then $T$ has exactly two minimal squares, a side square $S_{c}$ on $c$ and a vertex square $S_{C}$ at $C$, and $S_{C}$ is smaller than, equal to, or larger than $S_{c}$ precisely when $\gamma<45^{\circ}, \gamma=45^{\circ}$, or $\gamma>45^{\circ}$.

Proof. Since $h_{c} \geq c$, we conclude from Lemma 5 that $h_{a}<a$ and $h_{b}<b$, so $T$ has a side square $S_{c}$ on $c$ (whose side is $h_{c}$ ) and no other side squares. From Lemma 2 we see that $T$ has a vertex square $S_{C}$ at the vertex $C$ and at no other vertex. So $T$ has exactly two minimal squares, $S_{c}$ and $S_{C}$.

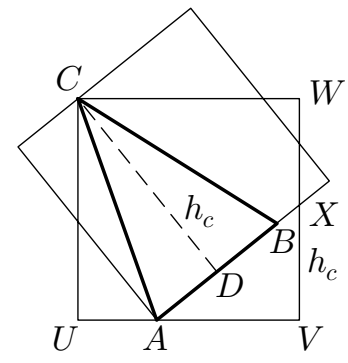

(a)

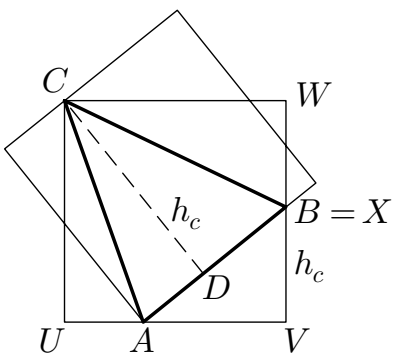

(b)

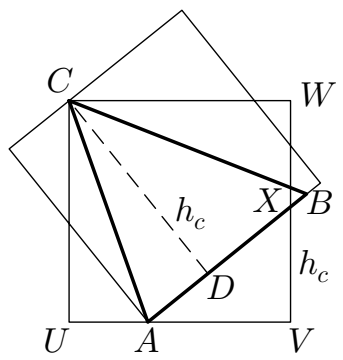

(c)

Fig. 5 Comparison of side and vertex squares

Let CUVW be a square with side $h_{c}$ positioned so that $A$ lies on $U V$ (Fig. 5). Let $D$ be the foot of the altitude from $C$ to $A B$, and let $X$ be the point where the ray $A B$ meets the side $V W$. Then since $\angle U C A=\angle A C D$ and $\angle D C X=\angle X C W, \angle A C X=$ $\frac{1}{2}(2 \angle A C D+2 \angle D C X)=\frac{1}{2}\left(90^{\circ}\right)=45^{\circ}$. Consequently $\gamma<45^{\circ}, \gamma=45^{\circ}$, or $\gamma>45^{\circ}$ according as $A-B-X, B=X$, or $A-X-B$. If $\gamma<45^{\circ}$ so that $A-B-X$ (Fig. 5(a)), then $S_{c}$ is not the smallest square that contains $T$ because a small motion would move $T$ inside $S_{c}$; and consequently $S_{C}$ must be smaller than $S_{c}$ in this case. If $\alpha=45^{\circ}$, then $B=X$ (Fig. 5(b)), and $S_{c}=S_{C}$ by the uniqueness assertion of Lemma 2. And if $\alpha>45^{\circ}$ so that $A-X-B$ (Fig. 5(c)) and if $S_{C}$ were smaller than $S_{c}$, then $T$ would fit in the larger square $S_{c}$ with the vertex $C$ at a corner, which it evidently does not do. 
The smallest square. Now it is easy to determine the smallest square that contains the given triangle. We continue to assume that the given triangle $T=A B C$ is positively oriented and $\alpha \geq \beta \geq \gamma$. In Fig. 6 (as in Fig. 4), $M D$ is the mediator of the longest side $B C$ of $T$, the circular arc $D B$ has center $C$, the segment $I K$ makes a $45^{\circ}$ angle with $B C$, and the circular arc $I J$ has center at the point $X$ for which $B M I X$ is a square.

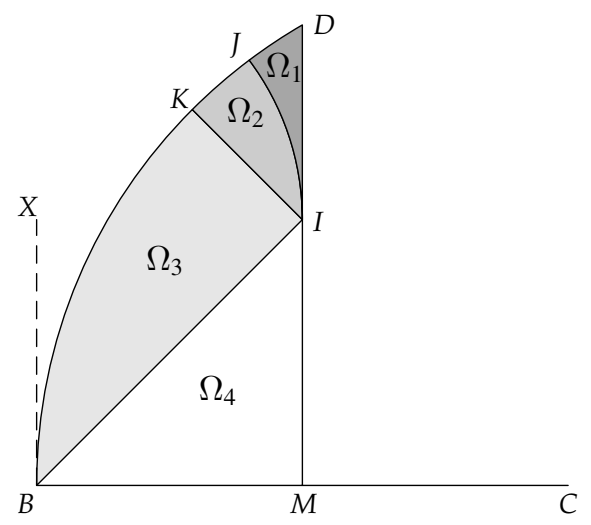

Fig. 6 The significant regions

The previous lemmas require four significant regions (Fig. 6). Recalling the normalization $a \geq b \geq c$, let

$$
\begin{aligned}
& \Omega_{1}=\left\{A: \beta, \gamma>45^{\circ}, \text { and } A X \geq a / 2\right\}, \\
& \Omega_{2}=\left\{A: \beta>45^{\circ}, \gamma \geq 45^{\circ}, \text { and } A X \leq a / 2\right\}, \\
& \Omega_{3}=\left\{A \neq I: \beta \geq 45^{\circ}, \text { and } \gamma \leq 45^{\circ}\right\}, \\
& \Omega_{4}=\left\{A: \beta \leq 45^{\circ}\right\},
\end{aligned}
$$

so that $\Omega_{1}$ is the closed circular triangular region $D J I$ minus the point $I, \Omega_{2}$ is the closed circular triangular region $K I J$ minus the point $I, \Omega_{3}$ is the closed circular triangular region $B I K$ minus the point $I$, and $\Omega_{4}$ is the closed triangular region $B M I$ (including $I)$. The point $I$, which represents the anomalous isosceles right triangle, is included in $\Omega_{4}$ but excluded from $\Omega_{1}, \Omega_{2}$, and $\Omega_{3}$.

Theorem 8 Let $T=A B C$ be a given positively oriented triangle labeled in such a way that $\alpha \geq \beta \geq \gamma$, and let the four regions $\Omega_{k}$ be defined by (4). Then the smallest square $S_{\min }$ that contains $T$ is

$$
S_{\min }= \begin{cases}S_{A} & \text { if } A \in \Omega_{1}, \\ S_{C} & \text { if } A \in \Omega_{2}, \\ S_{C} & \text { if } A \in \Omega_{3}, \\ S_{B C} & \text { if } A \in \Omega_{4},\end{cases}
$$


and $T$ fits into a square $S$ of side $s$ if and only if $s \geq s_{\min }$, where the side $s_{\min }$ of $S_{\min }$ is given by

$$
s_{\min }= \begin{cases}\frac{b c \cos \alpha}{\sqrt{b^{2}-2 b c \sin \alpha+c^{2}}} & \text { if } A \in \Omega_{1}, \\ \frac{a \sin \beta}{a b \cos \gamma} & \text { if } A \in \Omega_{2}, \\ \frac{\sqrt{a^{2}-2 a b \sin \gamma+b^{2}}}{\sqrt{2}} & \text { if } A \in \Omega_{3}, \\ \frac{a}{\sqrt{2}} A \in \Omega_{4} .\end{cases}
$$

Proof. See Fig. 6. If $A \in \Omega_{1}$, then $T$ is not tall by Lemma 6; it has just three minimal squares, a vertex square at each vertex by Lemma 2; and the smallest of these is $S_{A}$ by Lemma 3. The side of $S_{A}$ is given by (2). If $A \in \Omega_{2}$ or $\Omega_{3}$, then $T$ is tall by Lemma 6 . According to Lemma 7, it has just two minimal squares, a side square $S_{c}$ and a vertex square $S_{C}$, and the smaller is $S_{c}$ in $\Omega_{2}$ and $S_{C}$ in $\Omega_{3}$. The side of $S_{c}$ is $h_{c}=a \sin \beta$, and the side of $S_{C}$ is given by (2). Finally, if $A \in \Omega_{4}$ the diagonal square $S_{B C}$ is the only minimal square, and its side is $a / \sqrt{2}$.

We have finally arrived at an answer to our original question: The triangle with sides $a, b, c$ fits in the square of side $s$ if and only if $s \geq s_{\min }$, where $s_{\min }$ is given by (6). Theorem 8 also provides a solution for the dual problem: Given a square $S$ and a triangle $T_{0}$, find the largest triangle $T$ similar to $T_{0}$ that fits in $S$. Indeed, if $S$ has side $s$ and $T_{0}$ has sides $a_{0}, b_{0}$, and $c_{0}$, then $s_{0}=s_{\min }$ is the side of the smallest square that contains $T_{0}$, and the largest triangle $T$ similar to $T_{0}$ that fits in $S$ evidently has sides $s a_{0} / s_{0}, s b_{0} / s_{0}$, and $s c_{0} / s_{0}$.

\section{References}

[1] Post, K.A.: Triangle in a triangle: on a problem of Steinhaus. Geom. Dedicata 45 (1993), 115-120.

[2] Wetzel, J.E.: Squares in triangles. Math. Gazette 86 (2002), 28-34.

[3] Wetzel, J.E.: Rectangles in triangles. To appear in J. Geom.

Richard P. Jerrard

University of Illinois at Urbana-Champaign

Department of Mathematics

Urbana, IL 61801-2975, USA

e-mail: jerrard@uiuc.edu

John E. Wetzel

University of Illinois at Urbana-Champaign

Department of Mathematics

Urbana, IL 61801-2975, USA

e-mail: j-wetzel@uiuc.edu 